artelogie

\section{Artelogie}

Recherche sur les arts, le patrimoine et la littérature de l'Amérique latine

$12 \mid 2018$

Idiosyncrasie de l'indigénisme en Amérique latine.

Pluralité des sources et des appropriations extra-

latino-américaines

\title{
Kent Monkman : entre ré/appropriation et « Painting Back ».
}

\section{Valentina Pancaldi}

\section{(2) OpenEdition \\ Journals}

Édition électronique

URL : http://journals.openedition.org/artelogie/2704

DOI : 10.4000/artelogie.2704

ISSN : 2115-6395

Éditeur

Association ESCAL

Référence électronique

Valentina Pancaldi, « Kent Monkman : entre ré/appropriation et «Painting Back ». », Artelogie [En ligne],

12 | 2018, mis en ligne le 07 septembre 2018, consulté le 04 mai 2019. URL : http://

journals.openedition.org/artelogie/2704; DOI : 10.4000/artelogie.2704

Ce document a été généré automatiquement le 4 mai 2019.

Association ESCAL 


\title{
Kent Monkman : entre ré/ appropriation et « Painting Back ».
}

\author{
Valentina Pancaldi
}

«L'une des stratégies les plus courantes pour interpréter le présent est d'invoquer le passé. Pas seulement parce qu'on n'est pas d'accord sur ce qui a eu lieu alors, sur ce qu'a été le passé,

mais parce qu'on se demande si le passé est vraiment passé, mort et enterré, ou s'il continue, sous une forme différente peutêtre $»^{1}$

«En toute sérénité, je pense qu'il serait bon que certaines choses soient dites.

Ces choses, je vais les dire, non les crier. Car depuis

longtemps le cri est sorti de ma vie.

Et c'est tellement loin... » (FANON : 2001, 5) «Les nègres sont des sauvages, des abrutis, des analphabètes. [...]

Il y avait un mythe du nègre qu'il fallait démolir coûte que coûte » (Ibid. 95).

1 Si depuis l'Europe, le penseur et psychiatre martiniquais Frantz Fanon (1925-1961) calmement démontait et déconstruisait le mythe "blanc " du "nègre sauvage ", de l'autre côté de l'Atlantique, les Autochtones du Canada² démolissaient celui de l'« Indien Peau Rouge ".

2 Dès la « première rencontre » entre l'Europe et l'Amérique du Nord, et tout au long des cinq siècles suivants, dans l'inconscient collectif et l'imaginaire occidental, s'est consolidée une image folklorisée de l'Autochtone, c'est-à-dire celle d'un Indien "plumé » qui était à la fois noble et sauvage, mais aussi mourant et capable d'articuler seulement 
des cris ou des sons comme "Augh». Cette image a été véhiculée par toutes sortes de média et formes artistiques et notamment par la photographie, la peinture et le cinéma ${ }^{3}$.

La déconstruction critique de cet "Indien inventé " ${ }^{4}$ constitue, depuis quelques décennies, l'une des thématiques les plus exploitées par les nouvelles générations d'artistes autochtones. Parmi ces "Chasseurs / Chamanes / Guerriers de l'art » ainsi nommés par le sociologue et critique d'art, Guy Sioui Durand (2003 : 23), l'artiste multidisciplinaire Kent Monkman est l'une des voix la plus active et la plus originale.

Ontarien de naissance, mais d'ascendance crie (cree) et irlandaise, Kent Monkman est membre de la nation autochtone crie de Fisher River (Fisher River Cree Nation) située dans le nord du Manitoba ${ }^{5}$. En travaillant avec une variété de techniques différentes incluant peinture, film et vidéo, performance et installation, Monkman s'approprie la riche et protéiforme "imagerie indienne", en renversant les rôles et les codes, et propose ainsi une relecture subversive et humoristique de l'histoire qui ridiculise les " héros » européens et qui mine le récit conventionnel de la « découverte » du Nouveau Monde.

5 Sur le plan épistémologique, cet essai ambitionne d'établir un dialogue entre l'œuvre de Monkman et les études postcoloniales. L'application des théories postcoloniales aux arts, et plus précisément, aux littératures issues d'un contexte autochtone, a suscité de nombreuses réactions hostiles chez certains théoriciens et écrivains autochtones ${ }^{6}$. C'est notamment le cas de l'écrivaine Lee Maracle (1950-), membre de la nation Stó:lō, qui s'oppose fermement à l'usage de ces théories, car les conditions de vie des autochtones excluent toute possibilité de concevoir le "post-colonial $»^{7}$ et celui du romancier et essayiste d'origine cherokee, Thomas King, qui préfère garder sa distance envers le terme « postcolonial » bien que, comme il l'affirme, ne maitrise pas ces théories (KING, 1990 :10). Dans la plupart des cas, en fait, les résistances à l'application des théories dérivant des études postcoloniales dans le domaine artistique et littéraire autochtone, semblent-elles provenir d'un refus a priori de l'usage de toute épistémologie occidentale et/ou allochtone, mais surtout d'une interprétation inexacte du terme qui associe au préfixe "post» une valeur exclusivement temporelle et une fonction de césure avec le colonialisme.

Effectivement, on pourrait être tenté admettre que le terme "post-colonial » est très ambigu et peut générer de la confusion. Toutefois, les auteurs de Empire Writes Back ${ }^{8}$, texte fondateur des études littéraires postcoloniales, pensent autrement et donnent cette définition : « Nous emploierons toutefois le terme «post-colonial » pour recouvrir toute culture affectée par le processus impérial depuis le moment de la colonisation jusqu'à nos jours. Car il y a une continuité de préoccupations qui court tout au long du processus historique initié par l'agression impériale européenne » (ASHCROFT GRIFFITHS TIFFIN, $2012: 15) .9$

7 Par conséquent, le " post » n'implique pas la postérité en ce qui concerne le colonialisme ou le néocolonialisme, mais ce qu'en est un produit, ce qu'en dérive. Le terme "postcolonial » n'est pas un équivalent du terme « post-indépendance »; il n'indique pas « audelà » du colonialisme, mais plutôt « à partir du ». La notion de continuité employée par les auteurs nous suggère que les études postcoloniales se focalisent sur les effets discursifs et concrets dérivant du colonialisme dont encore aujourd'hui on en voit les résultats. On devrait considérer les études postcoloniales comme un ensemble de théories et de formulations liées à la production culturelle des populations colonisées « plutôt que comme une discipline ou une méthodologie en soi » (Ibid. : 225). La critique postcoloniale 
témoigne des forces inégales et inégalitaires de représentation culturelle qui sont à l'œuvre dans la contestation de l'autorité politique et sociale au sein de l'ordre mondial moderne (BHABHA, $2004: 245)$. Ces perspectives postcoloniales naissent du témoignage colonial des pays du Tiers-Monde et des discours des "minorités" dans le cadre des divisions entre l'Est et l'Ouest, le Nord et le Sud. Adopter une perspective postcoloniale signifie interroger les présupposés de l'époque coloniale, les effets de l'impérialisme et de la colonisation qui perdurent jusqu'à nos jours et opérer de manière récursive et subversive pour démanteler les idées reçues des théories européennes.

8 À partir de ce là il n'est guère étonnant de constater que les théories autochtones et celles postcoloniales partagent de nombreux éléments communs de réflexion critique tels que : les relations entre ascendance pure et hybridation culturelle et raciale, le (contre)discours de la représentation de l'« Autre » et de la résistance, la décolonisation des savoirs, la révision de l'historiographie et les conséquences de la cohabitation entre colonisateurs et colonisés. D'autant plus, les théories concernant la dépossession territoriale et les archives culturelles de l'Occident formulées par Edward Saïd, consolident le lien entre le discours postcolonial et celui autochtone. C'est donc cette proximité qui nous autorise à créer un dialogue entre ces deux épistémologies.

9 Comme nous le suggère le titre, le but de cet essai sera d'interroger l'œuvre de Kent Monkman à partir d'une notion pivot des études postcoloniales, à savoir le «Writing back ». Dans le cadre des théories littéraires postcoloniales, cette notion renvoie à la réécriture des œuvres canoniques occidentales. C'est une opération de renversement épistémologique considérable, car elle implique une (ré)appropriation et une subversion du canon littéraire occidental. En tenant compte du fait que presque la totalité des œuvres de Monkman contiennent des citations plus ou moins directes du canon pictural occidental, dans quelle mesure peut-on alors poser que cet artiste participe au Painting back?

10 À ce point de notre réflexion, un approfondissement de la notion de «Writing Back » s'impose.

\section{Qu'entend-on par « Painting Back »?}

11 Pour répondre à cette question, il nous faut faire un détour épistémologique par le terme «Writing back ». Selon la théorie postcoloniale, il recouvre deux acceptions : stricto sensu, il s'agit du fait qu'une œuvre prend comme point de départ une autre œuvre (ou quelques éléments de cette œuvre) appartenant à la tradition canonique occidentale pour la relire, récrire, réinterpréter et la remettre en question ${ }^{10}$. L'auteur, issu dans la plupart des cas d'un contexte de colonisation, engage un dialogue avec l'œuvre canonique (et le canon lui-même) pour en contester l'autorité et la revoir et en proposer ainsi un point de vue inédit et alternatif ${ }^{11}$.

12 Lato sensu, on indique le geste de cette réponse, de cette " contre-attaque créative » de la part des auteurs envers le canon occidental, donc le canon des colonisateurs. Dans l'expression writing back, le mot «back» n'est pas neutre, ne signifie pas «vers » ou " pour » le centre, mais « contre » les prétentions affirmées par le centre à la légitimité et au pouvoir (ASHCROFT GRIFFITHS TIFFIN, $2012: 47$ ).

13 En mélangeant différentes formes et techniques littéraires telles que la parodie, la satire, le pastiche linguistique, ces auteurs érigent un contre-discours qui mine l'hégémonie des 
œuvres « canoniques » européennes. On peut considérer ce processus de réappropriation et de subversion du canon comme un geste de résistance et de décolonisation culturelle. Comme le souligne le théoricien palestinien Edward Saïd (1935-2003) « loin d'être une simple réaction à l'impérialisme, [cette résistance] est une conception alternative de l'histoire humaine " (SAÏD, $2000:$ 308). Le writing back est donc un moyen qui sape l'historiographie européenne : le sujet marginalisé, réduit en silence et privé de son agentivité reprend la parole et nous raconte l'Histoire de son point de vue.

La transposition de cette notion de writing back du plan littéraire vers le plan pictural nous donne le «Painting Back». Autrement dit, le painting back est à la peinture canonique ce qu'est le writing back à la littérature canonique. En effet, le painting back vise à subvertir le canon pictural des colonisateurs, à nuancer les critères esthétiques de l'« universalité européenne", à se moquer des clichés sur l' "Autre» afin de détourner ses fausses représentations et, finalement, à peindre l'histoire d'un autre point de vue.

Dans le contexte autochtone, il s'agit non seulement de déconstruire le mythe de l'« indien inventé ", mais surtout de peindre la réelle violence coloniale présente dans l'œuvre impérialiste.

\section{L'œuvre de Kent Monkman : de la dépossession à la "ré/appropriation ».}

16 La terrible crise d'Oka entre la nation Mohawk de la Reserve Kahnawake et les forces armées canadiennes $(1990)^{12}$, le $500^{\text {ème }}$ anniversaire de la « découverte » des Amériques (1992) et le Sommet de la Terre à Rio (1992) ont déclenché la naissance de nouvelles formes de résistance artistique et un changement de paradigme : "à la dépossession », les artistes autochtones ont répondu à travers « une réappropriation » (SIOUI DURAND 2003 : 30), c'est-à-dire avec une récupération et une actualisation des savoirs et codes culturels ancestraux. Monkman non seulement réalise une réappropriation du réservoir culturel autochtone, mais il s'approprie également les codes occidentaux afin de les subvertir. Par cette "ré/appropriation", Monkman vise à déconstruire les représentations déformées des Autochtones et il nous montre comment l'histoire est en réalité, un processus subjectif qu'il faut interroger de façon continue.

Chez Monkman, l'humour joue un rôle central. Son ironie est connotée d'une forte charge critique qui provoque le rire et soudainement invite le public à réfléchir sur sa propre perception à l'égard des cultures autochtones amérindiennes. Comme le souligne justement Jonathan Lamy Beaupré, «c'est avec humour et par l'humour » (2012:12) qu'il démonte les stéréotypes. L'humour est à la fois déclencheur et vecteur de la démystification iconographique de l' "indien inventé », et dans cette démarche, Monkman n'est pas tout seul.

Il a créé un alter ego : «Miss Chief Share Eagle Testickle », le résultat vivant et grotesque $\mathrm{du}$ croisement de tous les stéréotypes regardant les Autochtones. Cet alter ego tienne une place prépondérante dans l'œuvre de Monkman: elle apparait non seulement dans la plupart des peintures et des installations de l'artiste, mais aussi dans ses performances et ses courts-métrages où Miss Chief n'est incarnée par nulle autre que Kent Monkman luimême. Miss Chief Share Eagle Testickle est le résultat pop, glamour et queer d'un mélange kaléidoscopique inspiré premièrement par les images des "indiens" véhiculées par l'industrie cinématographique hollywoodienne et l'art pictural du XIX siècle ${ }^{13}$, et 
deuxièmement par la chanteuse Cher, en particulier pendant la phase de son succès obtenu grâce à sa chanson «half-breed »" ${ }^{14}$. En créant et en incarnat son alter ego, Kent Monkman symbolise les stéréotypes de l'imaginerie «indienne» pour les parodier, les ridiculiser, et les dénoncer.

Avec ses talons vertigineux, son mascara généreux, son carquois de Louis Vitton, son plumage excessif et ses vêtements à paillettes très succincts, cette excentrique "postindian diva warrior» (LISS, MADILL, 2008: 31) dénonce l'assujettissement des populations autochtones, leur manipulation médiatique, et notamment l'impact que la colonisation et le christianisme ont eu sur leur éducation et leur sexualité.

En se servant du personnage de Miss Chief, Monkman opère de fait la récupération du «Two Spirit $»^{15}$, une personne incarnant l'existence de l'esprit mâle et femelle dans un seul corps qui existait au sein des communautés amérindiennes de l'Amérique du Nord et que les missionnaires chrétiens ont essayé d'éradiquer en raison de son offense à la pudeur de la morale catholique.

21 On peut considérer Miss Chief comme la clé d'interprétation de toute l'œuvre artistique de Monkman et sa parfaite mise en abîme. Elle échappe à toute tentative de définition fixe, car elle-même symbolise le refus d'une fixité. Elle reflète la pensée de Monkman qui est profondément anti-dualiste et anti-dichotomique. Dans le sillage du Trickster, mais aussi du Two Spirit, Miss Chief s'oppose à toute sorte de binarisme de genre et d'identité. Elle brise toute convention «occidentale» laissant ainsi le spectateur «blanc» sans aucun repère familier et provoque chez lui un dépaysement, plus précisément, une défamiliarisation. La phénoménologie de Miss Chief nous invite à une expérience identitaire. Tantôt, elle capture et monopolise notre attention en provoquant le rire, tantôt soudain, elle nous invite à réfléchir plus profondément et à nous interroger sur notre perception de la manière autochtone d'« être au monde ».

La force esthétique et catalysatrice dont dispose le personnage Miss Chief lui permet de se traduire dans toutes les formes artistiques déployées par Monkman, sans que son message porteur de dénonciation de l'oppression subie par les populations autochtones soit altéré. Peu importe qu'elle soit la position occupée par Miss Chief lors d'une installation, une performance ou dans une peinture, elle constitue toujours le point focal de la scène et, ce faisant, de notre attention.

L'œuvre artistique qui plus célèbre cette héroïne est la peinture The Triumph of Mischief (2007) [fig.1], une véritable apothéose des éléments les plus récurrents chez Monkman, parmi lesquels l'appropriation de topoi occidentaux.

Cette peinture, en fait, pullule de "pillages", empruntés au canon occidental, et davantage, de la période allant du Baroque européen au Romantisme américain. Bien entendu, il ne faut pas penser ces citations comme un hommage, mais plutôt comme une réponse, car elles ne sont ni neutres, ni dénuées de sens.

Le cas le plus éclatant concerne le paysage : le fond de toile de The Triumph of Mischief correspond exactement à celui de Looking up the Yosemite Valley (1865-1867) [Fig. 2] du paysagiste américain Albert Bierstadt (1830-1902). Dans la majorité de ses toiles, en parfaite syntonie avec le mythe de l'« errand into the wilderness $»^{16}$, l'Ouest américain est représenté comme un territoire très peu civilisé, vierge, à explorer, voire occuper, tandis que les Autochtones sont souvent situés aux marges ${ }^{17}$. Dans Looking up the Yosemite Valley, les Autochtones ne sont même pas représentés, malgré le fait que ce territoire appartient aux populations Ahwahnee ${ }^{18}$. Dans ce cas, Monkman s'approprie une peinture canonique 
et son paysage censuré pour revendiquer la propriété du territoire et le restituer aux réels possesseurs. Comme il l'affirme au cours d'une interview avec Cathy Mattes : « I felt that borrowing from their landscape paintings would be a way of reclaiming some of the land they had stolen from us. Their version of history was one-sided " (LISS, MADILL, 2008 : 108). Ce geste de revendication et de réappropriation du territoire accompli par Monkman, se veut une prise de position contre les discours coloniaux et impérialistes et, en termes saïdiens, un acte de "réinscription ».

Selon Saïd, l'impérialisme est tout d'abord une violence géographique, une : «visée, installation et mainmise sur une terre qu'on ne possède pas, un territoire lointain où d'autres vivent et qui leur appartient. Pour toutes sortes de raisons, cette perspective séduit certains et implique souvent pour d'autres des malheurs sans nom » (SAÏD, 2000 : 41). ${ }^{19}$ Cet acte de dépossession: " par lequel la quasi-totalité de l'espace mondial est exploré, cartographié et finalement annexé » signe le début de l'asservissement colonial, au détriment de l'indigène, qui assiste à la perte de son espace au profit de l'étranger ( Ibid. : 320).

La «réinscription" est donc une forme de résistance culturelle à cette violence géographique impérialiste. Elle est une stratégie décolonisatrice qui vise à la restauration de l'identité géographique du dépossédé. Le colonisé, selon Saïd, ne perd pas seulement l'accès physique à ses lieux, il en est aussi dépossédé aussi dans son imaginaire. L'identité géographique, surtout dans le cas d'une présence définitive du colonisateur sur le territoire, n'est récupérable que par l'imagination, les arts et la littérature (Ibidem) ${ }^{20}$.

Se réinscrire ${ }^{21}$ (dans les territoires) signifie reprendre possession du discours sur le territoire, renommer les cartes, épurer la toponymie impérialiste, mais surtout se réapproprier des lieux par la littérature et les arts, comme dans le cas de Monkman.

Sur plan épistémologique, cette réinscription s'intensifie avec l'insertion des Autochtones dans le paysage censuré de Bierstadt. Dans The Triumph of Mischief, Monkman se réapproprie non seulement le territoire, mais surtout il réécrit l'Histoire du point de vue des Autochtones. Comme Monkman l'explique: «In the Romantic period, we were painted out of the narratives [...]. The reason I respond to these artists is because I think their work is important. It's worth examining that whole period of art, so purely onesided, like a big cover-up of what was really happening. I try to approach it with humour [...] it's a very gentle way of making people aware of this huge obliteration of our narratives » (MONKMAN, 2012) .

30 La période artistique du Romantisme américain qui glorifiait la nature sauvage et, de fait, excluait les Autochtones, doit donc être préservée comme patrimoine à contester et à déconstruire. Il s'agit de faire apparaitre les traces de la dépossession, y mettre l'accent, pour les contester et les déjouer.

31 En déplaçant le regard sur les personnages présents dans l'œuvre, on notera que Miss Chief est, immanquablement, au centre de la scène. Sa présence, en tant que porte-parole de l' " être au monde " autochtone, est aussi un acte de prise de parole contre la mise en silence opérée par les discours impérialistes ${ }^{22}$.

Miss Chief domine une scène (homo)érotiquement très chargée, fidèle au style « Camp »" où quarante-sept figures de toutes les époques et de tous les lieux évoquent une situation typique des Bacchanales. Le châle rose entourant Miss Chief, la présence de figures mythologiques telles que les satyres, le silène, le Minotaure, le dieu Pan et le choix même du titre semblent être des allusions à l'œuvre Il trionfo di Bacco e Arianna (1577-1578) 
[fig.3], du peintre italien Annibale Caracci (1560-1609). Dans ce dernier les trois putti, en parfaite continuité avec la tradition de la Renaissance renvoient à l'amour, alors que chez Monkman ils représentent autre chose : ils symbolisent l'impact du christianisme sur la sexualité autochtone (SWEET : 2017).

La réponse autochtone à cette violence de la morale chrétienne ne se fait pas attendre. En derrière-plan, juste avant le petit lac, on notera un Autochtone sur un cheval gris sodomisant un moine. Juste à droite, Monkman insère une autre scène de sodomie : un Autochtone est surpris à insérer des fleurs dans le postérieur du général américain George Custer. Il s'agit d'une appropriation d'un détail similaire du panneau central du triptyque Le Jardin des délices (1494-1505) [fig. 4] de Jérôme Bosch (1450-1516) où un jeune homme peut être observé en insérant des fleurs dans le postérieur d'un autre homme.

À droite de Miss Chief, Pablo Picasso est entouré de ses « demoiselles » d'Avignon (1907) et tient dans les mains un masque africain. Le chef de file du Modernisme est à contrecœur poussé devant Miss Chief et semble d'être jugé par Monkman au sujet de ses " crimes ", c'est-à-dire l'appropriation et la décontextualisation de l'art tribal africain ${ }^{24}$. À notre avis, Monkman, en parodiant Picasso et en s'inscrivant dans le sillage de l'artiste Jean-Michel Basquiat, semble condamner le mouvement artistique dénommé "Primitivisme", né au début du XXe siècle sous-tendant justement l'intérêt, voire la fascination, envers les objets et les œuvres d'art issues des cultures autochtones, dites "primitives $»^{25}$.

En suivant le regard de Picasso, on tombe sur un homme nu donnant le dos au spectateur. Il s'agit d'un guerrier roman tiré de L'intervention des Sabines (1799) [fig.5] du peintre français Jean Louis David (1748-1825). Monkman décontextualise le soldat son époque romaine pour le transporter dans l'époque de la Conquête des Amériques : il ne porte plus la galea, le casque romain typique des légionnaires, mais le casque des Conquistadores espagnols. De plus, l'artiste « indigénise » ce légionnaire-conquistador en lui substituant le bouclier et les sandales romaines avec un tambour et des mocassins autochtones.

Les trois danseurs situés juste derrière le légionnaire-conquistador-autochtone font allusion aux «Indiens » Tlingit de Medicine Mask Dance (1848) [Fig.6] du peintre irlandais Paul Kane (1810-1871). Toutefois, en regardant plus attentivement, on notera que la couleur de leur peau est blanche et cela pourrait nous indiquer qu'il s'agit donc d'Européens ou plus généralement, d'Occidentaux. Il se peut que Monkman ait voulu inverser les rôles afin de représenter (et se moquer) des «Blancs qui jouent aux Indiens ». D'ailleurs, la scène entre le centaure et l'« indien » à cheval pourrait renforcer cette thèse du renversement des rôles. Ce dernier, habillé d'une veste rouge similaire à celle des colons européens, brandit un lasso et tente de capturer une figure mythique "sauvage " de la tradition occidentale. On pourrait donc l'interpréter comme un Indien jouant à faire le blanc, ou bien le cowboy.

Ce renversement de rôles, de stéréotypes et de points de vue est une dialectique qui revient souvent chez Monkman, mais elle atteint son paroxysme dans Artist and the Model (2007) [Fig.7]. Ici, notre artiste bouleverse toute dynamique de pouvoir entre sujet et objet, colonisé et colonisateur. Il s'approprie, en fait, l'œuvre Painting the Portrait of Mahto-toh-pa-Mandan [Fig. 8] (1861-1869) de George Catlin (1796-1872) pour la subvertir et en contester l'autorité «canonique ». Ce qui en résulte est une œuvre méta-artistique très puissante. Dans un paysage évoquant celui de Chinook Indians in Front of Mount Hood [fig.9] (1851-1856) de Paul Kane, Miss Chief, voluptueuse comme Cher et portant une coiffe de plumes et un arc plutôt « stylé », remplace Catlin pour peintre « son objet ». 

cowboy sui generis. Comme le suggère Richard Hill, son visage ressemble à celui de James Dean incarnant Jett Rink dans le film Giant (1956), mais son corps renvoie à l'esthétique pornographique gay «camp» du dessinateur et photographe finlandais Tom of Finland (THERIAULT, $2012: 80$ ). 11 est fortement probable, à notre avis, qu'il agisse d'Edward Curtis (1868-1952), à savoir le photographe ethnologue américain qui a été l'un des premiers à « inventorier » les Autochtones nord-américains et à répandre le mythe de la «Vanishing Race». La parodie de Curtis, de ses photos symbolisant «les Indiens mourants» et la dénonciation de l' «ethnographie de sauvetage» ce sont des thématiques qui reviennent très souvent dans l'oeuvre de Monkman ${ }^{26}$ et dans celles des autres artistes autochtones de l'Amérique du Nord ${ }^{27}$. Pas loin de ses pieds on remarquera un dernier indice qui nous suggère la réelle profession de ce cow-boy épinglé et, ainsi nous confirme notre thèse : une caméra frappée par un tomahawk. Voilà encore une autre revendication de la part d'une "race bien plus que vivante " contre les sciences européennes dites «de sauvetage». Un autre bouleversement s'accomplit: c'est Miss Chief, porte-parole d'une « race vivante », qui inventorie ici un sujet mourant. Chief lui tire, on remarquera qu'à l'instar de l'ethnographie européenne, elle classifie cette espèce d'homme européen ${ }^{28}$. Lors d'une interview-performance, Monkman-Miss Chief a déclaré « They [les hommes européens] treated me like an object... which I'm not ! [...] I really got bored of that quickly. So I decided that it was time to turn the tables and be the artist, and not the model » (LISS, MADILL, $2008: 107$ ). Le bouleversement est donc plus qu'accompli, cette fois-ci c'est elle qui, au nom des Autochtones, représente et ridiculise.

Lors de la susdite interview-performance, à la question : « What else is your motivation in painting ? ", Monkman-Miss Chief a-t- elle ainsi répondu : "Well, the Europeans in North America, they had pillaged our society. They had stolen our land. They created this whole document called "art history" found their exploits here in America I also wanted to reference that » (Ibid. :109).

41 La relecture des artistes occidentaux opérée par Monkman s'inscrit dans une logique en contrepoint, selon laquelle observer et interpréter une ouvre signifie premièrement la considérer une réalité protéiforme, quelquefois nourrie du point de vue historique d'une expérience coloniale, et deuxièmement prendre en compte toutes sortes de "pratiques spatiales où géographiques et rhétoriques - accents, limites, contraintes, intrusions, inclusions, interdits - qui toutes contribuent à élucider une topographie [mondiale] complexe et inégale » (SAÏD : 2000, 441). Monkman relit la tradition artistique occidentale qui de fait marginalisait, déclarait mourants et censurait les Autochtones, et s'approprie codes, topoi, et formes afin de lui adresser un contre-discours.

$\mathrm{Au}$ début de cet article, nous nous sommes demandé si peut-on considérer l'œuvre artistique de Monkman un painting back. L'affirmer ce serait une erreur, le nier serait une erreur également. Pour répondre à notre question : oui, l'œuvre de Monkman est aussi un painting back. Malgré le fait que l'appropriation des topoi occidentaux, la réponse au colonisateur et la réécriture de l'Histoire occupent une place fondamentale dans l'œuvre de Monkman, soutenir que toute l'œuvre de Monkman est un painting back serait trop réducteur, car elle s'inscrit dans une démarche qui est beaucoup plus vaste, plus fluide et plus riche. 
L'art de Monkman, en fait, se pose comme le lieu d'un dialogue non hiérarchisé qui récuse toute binarité, fixité, altérité, s'offrant à son public comme expérience polysémique, collective, inclusive et ironique. De tangente multimédiale, son art s'exprime par l'alter ego, Miss Chief, qui est à la fois clé et mise en abyme de toute l'œuvre de Monkman. En traversant le temps et l'espace géographique et en franchissant les frontières, qu'elles soient de genre, religieuses, culturelles, sociales, et ethniques, Miss Chief devient porteuse d'un message contraire à tout dogmatisme et essentialisme. Penser en contrepoint aux autres, comme le soulignait Saïd, implique de ne pas chercher à dominer, étiqueter, et surtout d'arrêter de penser à «notre » culture, «notre " pays, en termes exclusifs et exclusivistes (SAÏD : 2000, 464). Une telle ouverture pourrait prévenir tout dogmatisme enfermant la pensée des «Blancs » d'un côté et celle des Autochtones de l'autre.

« Nul aujourd'hui n'est seulement ceci ou cela. Indien, femme, musulman, américain, ces étiquettes ne sont que des points de départ. Accompagnons ne serait-ce qu'un instant la personne dans sa vie réelle et elles seront vite dépassées. L'impérialisme a aggloméré à l'échelle planétaire d'innombrables culture et identités. Mais le pire et le plus paradoxal de ses cadeaux a été de laisser croire aux peuples qu'ils étaient seulement, essentiellement, exclusivement, des Blancs, des Noirs, des Occidentaux, des Orientaux. Comme ils font leur histoire, les êtres humains font aussi leurs cultures et leurs identités ethniques. Les continuités persistantes sont indéniables : longues traditions, habitats prolongés, langues nationales, géographies culturelles. Mais il n'y a aucune raison, sauf la peur et le préjugé, de vouloir à toute force les maintenir séparées et distinctes, comme si c'était le fin mot de la vie humaine. En fait la survie dépend des liaisons entre les choses; on ne peut priver la réalité, dit Eliot, des « autres échos [qui] habitent le jardin » (Ibidem).



Fig.1The Triumph of Mischief, Kent Monkman, 2007, 213 × 335 cm, acrylique sur toile, Musée de Beaux-Arts du Canada, Ottawa (Canada).

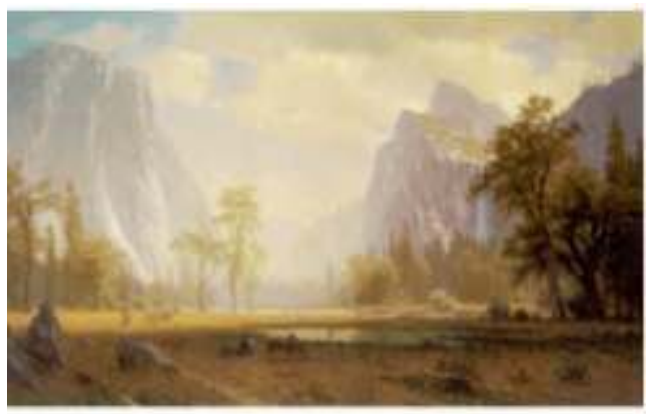

Fig.2 Looking up the Yosemite Valley, Albert Bierstadt, 1865-1867, $91.4 \times 148.6 \mathrm{~cm}$, huile sur toile, Haggin Museum, Stockton, (California). 


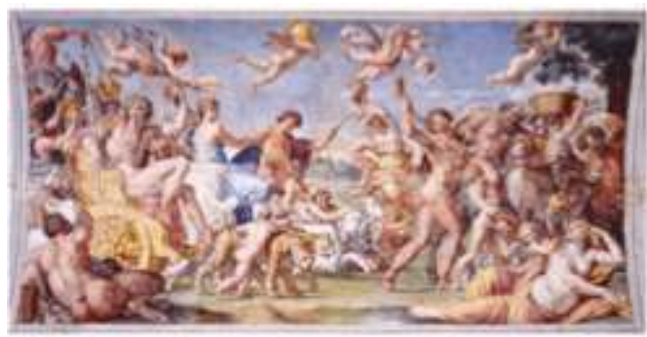

Fig.3 II trionfo di Bacco e Arianna, Annibale Caracci, 1577-1578, fresque, Palazzo Farnese, Rome (Italie).

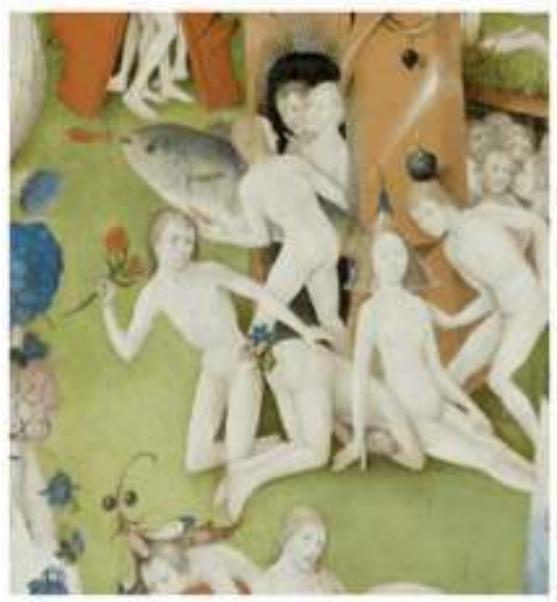

Fig.4 Le Jardin des délices, Jérôme Bosch, 1494-1505, 220 × 390 cm, huile sur toile, Musée du Prade, Madrid (Espagne), détail.

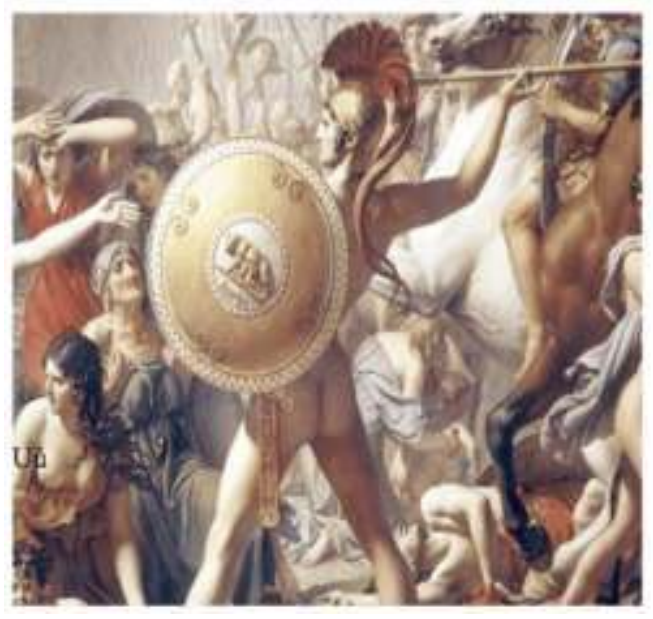

Fig.5 Les Sabines, Jacques Louis David, 1799, 385 x 522 cm, huile sur toile, Musée du Louvre, Paris (France) détail. 


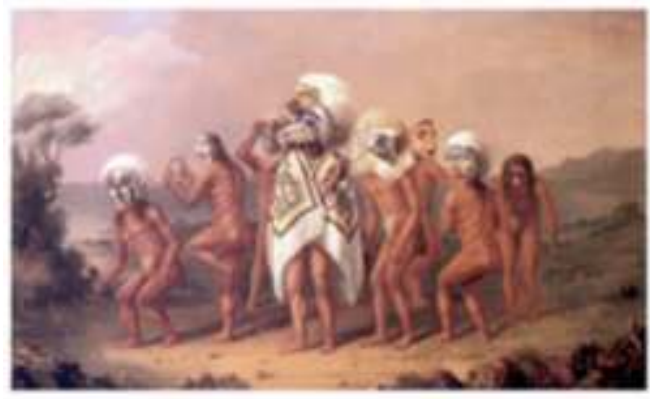

Fig.6 Medicine Mask Dance, Paul Kane,1848, huile sur toile, Royal Ontario Museum, Toronto (Canada).

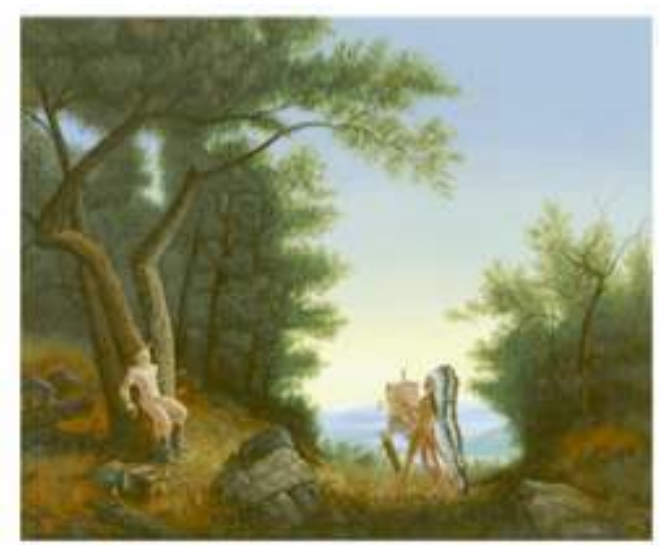

Fig.7 The Artist and the Model, Kent Monkman, 2007, 50 × $60 \mathrm{~cm}$ acrylique sur toile, Collection de l'artiste

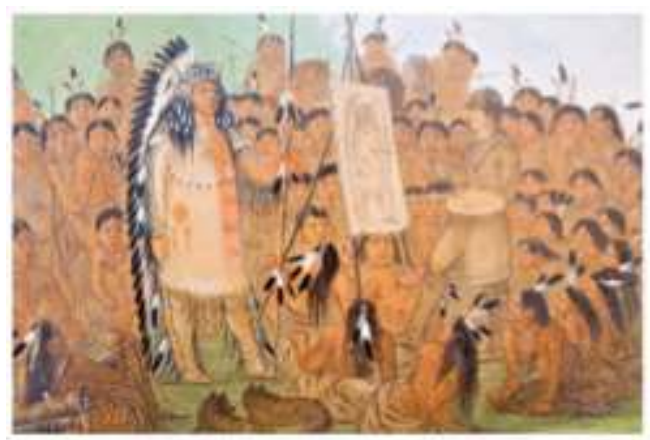

Fig.8 Painting the Portrait of Mah-to-toh-pa-Mandan, George Catlin, 1861-1869, $47 \times 62 \mathrm{~cm}$, huile sur papier, National Gallery of Art, Washington, (États-Unis), detail.

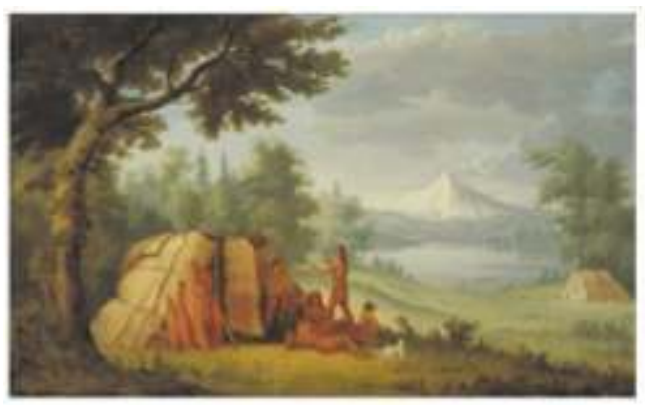

Fig. 9 Chinook Indians in Front of Mount Hood, Paul Kane, 1851-1856, 46 x 74 cm, huile sur toile, Musée de Beaux-Arts du Canada, Ottawa (Canada). 


\section{BIBLIOGRAPHIE}

BILL Ashcroft, Gareth GRIFFITHS et Helen TIFFIN, L'Empire vous répond : Théorie et pratique des littératures post-coloniales, Bordeaux, Presses Universitaires de Bordeaux, 2012.

BHABHA Homi K., The Location of Culture, Londres-New York, Routledge, 2004.

FANON Frantz, Peau noire, masques blancs, Paris, Éditions du Seuil, 1952.

HERAULT Laurence, « Transgression et désordre dans le genre : les explorateurs français aux prises avec les “ berdaches " amérindiens ", Etnográfica, vol. XIV, n², 2010. [URL] http:// etnografica.revues.org/316

KING Thomas, « Godzilla vs. post-colonial », World Literature Written in English, Vol.30, n²2, pp.10-16, 1990. [URL] http://dx.doi.org/10.1080/17449859008589128

LAMY BEAUPRÉ Jonathan, Du stéréotype à la performance : les détournements des représentations conventionnelles des premières nations dans les pratiques performatives.

Thèse en sémiologie, soutenue en Mai 2012, Université du Québec à Montréal, co-dirigée par Simon Harel et Josette Féral.

LEGGATT Judith « Native Writing, Academic Theory : Post-colonialism across cultural divide » dans Is Canada Postcolonial ? Unsettling Canadian Literature, sous la direction de Laura MOSS, Waterloo, Wilfrid Laurier University Press, 2003.

LEGROS Dominique, «Les Demoiselles d'Avignon de Picasso et les pièces africaines contemporaines du tableau : Esthétique émancipatrice ou répressive ? », Sociologie et sociétés, $\mathrm{n}^{\circ}$ 172, 1985. [URL] http://id.erudit.org/iderudit/001426ar

LISS David et MADILL Shirley, The Triumph of Mischief: Kent Monkman, Hamilton, éd. Art Gallery of Hamilton ABC, 2008.

MARACLE Lee, « The "Post-Colonial" Imagination ", dans Unhomely States: Theorizing EnglishCanadian Postcolonialism, sous la direction de Cynthia SUGARS, Toronto, Broadview Press, 2004.

MENARD Guy, « Du berdache au Berdache : lectures de l'homosexualité dans la culture québécoise » Anthropologie et Sociétés, n 93, 1985, pp.115-138. [URL] http://www.erudit.org/fr/ revues/as/1985-v9-n3-as509/006292ar

MONKMAN Kent, « Tonto Takes Charge. Miss Chief Eagle Testickle : a portrait », The Warlus Arts and Culture, 2012. [URL] https://thewalrus.ca/2008-05-detail/

POLAK Iva, « Postcolonial Imagination and Postcolonial Theory : Indigenous Canadian and Australian Literature Fighting for (Postcolonial) Space ", Theory and Practice in English Studies, $\mathrm{n}^{\circ} 4$, 2005. [URL] http://www.phil.muni.cz/plonedata/wkaa/Offprints\%20THEPES\%204/TPES\%204\%20 (135142)\%20Polak.pdf

SAÏD Edward Wadie, Culture et Impérialisme, Paris, Fayard-Le Monde Diplomatique 2000 (Version anglaise : Culture and Imperialism, New York, Vintage Books, 1993).

SIOUI DURAND Guy, "Jouer à l'Indien est une chose, être Amérindien en est une autre ", Recherches amérindiennes au Québec, Vol. XXXM, N³, 2003, p. 23.

SONTAG Susan, Against interpretation and other essays, Londres, Penguin, 2009. 
SWEET Kevin, « Le peintre Kent Monkman revoit l'histoire du Canada », Ici Radio Canada [URL] http://ici.radio-canada.ca/nouvelle/1013000/le-peintre-kent-monkman-revoit-lhistoire-ducanada

THÉRIAULT Michèle (dir.), Interpellations : three essays on Kent Monkman / trois essais sur Kent Monkman, Montréal, éd. Galerie Leonard \& Bina Ellen Art Gallery, à Concordia University, 2012.

THIEME John, Postcolonial Con-texts: Writing Back to the Canon. Literature, Culture and Identity, Londres, Continuum Press, 2001.

TUHIWAI SMITH Linda, Decolonizing Methodologies, Research and Indigenous Peoples, Palgrave, University of Otago Press, 2001.

\section{NOTES}

1. Edward W. Saïd, Culture et Impérialisme (traduction de Culture and Imperialism, New York, Vintage Books, 1993) Paris, Fayard-Le Monde Diplomatique 2000, p.37. Dans cet article toutes les citations seront tirées de l'édition française.

2. Dans cet article, on emploiera le terme «Autochtones" pour faire référence à toutes les populations amérindiennes autochtones nord-américaines. Nous privilégions cette définition, qui est la plus utilisée dans le Canada francophone, puisqu'elle englobe les membres des Premières Nations, les Inuits et leurs descendants.

3. Il suffit penser au cinéma Western qui a été, selon ses phases, un vrai réservoir de stéréotypes concernant les Autochtones. Parmi les plus exploités, on trouve celui de «l'indien noble sauvage », du « diable rouge féroce et sanguinaire » ou encore de "l'indien naîf et naturaliste ». Ce dernier a en outre préfiguré le stéréotype de «l'Indien New Age » qui a dominé le cinéma hollywoodien des années '70.

4. Pour un approfondissement de la notion d'« Indien inventé » voir James Clifton, The Invented Indian: Cultural Fictions \& Government Policies, New Brunswick, Transaction Publishers, 1990, et Jean-Jacques Simard, La réduction : l'autochtone inventé et les amérindiens aujourd'hui, Québec, Septentrion, 2003

5. Il naît en 1965 à St. Mary's, en Ontario, mais grandit à Winnipeg et dans diverses réserves autochtones situées dans le nord de l'état du Manitoba. Cette enfance itinérante est due à son père : il était prêcheur chrétien et bilingue, en mission d'évangélisation auprès des réserves autochtones du peuple Cris. Ses œuvres ont été présentées dans le cadre d'expositions personnelles un peu partout au Canada y compris le Musée des Beaux-Arts de Montréal, le Musée d'Art Contemporain Canadien à Toronto et la Winnipeg Art Gallery. Il a également participé à différentes expositions collectives internationales et ses courts-métrages ont été présentés dans de nombreux festivals nationaux et internationaux, y compris la Berlinale $(2007,2008,2015)$ et le Toronto International Film Festival (2007). Dans la sphère publique, ses œuvres sont exposées de façon permanente au Musée des Beaux-Arts du Canada à Ottawa et au Musée des Beaux-Arts et au Musée d'Art Contemporain de Montréal. Il a un site web personnel : http:// www.kentmonkman.com/

6. Voir à ce sujet Judith Leggatt, « Native Writing, Academic Theory : Post-colonialism across cultural divide ", Is Canada Postcolonial ? Unsettling Canadian Literature, Waterloo, Wilfrid Laurier University Press, 2003, pp. 111-126 ; Linda Tuhiwai Smith, Decolonizing Methodologies, Research and Indigenous Peoples, Palgrave, University of Otago Press, 2001. Surtout les pages 14, 24, 98 ; Iva Polak, « Postcolonial Imagination and Postcolonial Theory : Indigenous Canadian and Australian Literature Fighting for (Postcolonial) Space » Theory and Practice in English Studies, n 4, 2005, [URL] 
http://www.phil.muni.cz/plonedata/wkaa/Offprints\%20THEPES\%204/TPES\%204\%20(135142)\% 20Polak.pdf [consulté le 18/04/2017]

7. Elle a affirmé : "With conditions as they are, it is a luxury for me to wander into my dreamspace and conceive of "post-colonial." A multitude of faces, all white and too numerous to name, gather around the edges of my dreamspace. [...].». Voir Lee Maracle "The "Post-Colonial" Imagination " Unhomely States : Theorizing English-Canadian Postcolonialism, dir. Cynthia Sugars, Mississauga, Broadview Press, 204-8, p.205. Voir de la même auteure « Native Myths: Trickster Alive and Crowing ", Fuse n' 29, 1989 p.185.

8. Le titre de cet ouvrage fait référence à l'article "The Empire Writes Back with a Vengeance » de Salman Rushdie, publié le 3/07/1982 dans le journal britannique The Times. Toutes les citations qui suivent seront tirées de la version traduite en français: L'Empire vous répond: Théorie et pratique des littératures postcoloniales, Presses Universitaires de Bordeaux, Bordeaux 2012.

9. Autour de la définition du terme " post-colonial » et de l'usage du trait d'union a été soulevé un vrai débat qui a vu la participation de plusieurs théoriciens et intellectuels. Pour en savoir plus voir V. Mishra e B. Hodge, What is Post(-) colonialism?, P. Williams and L. Chrisman, Colonial Discourse and Post-Colonial Theory A Reader, New York, Columbia University Press, 1993. Ici on fera référence à la définition donnée par Ashcroft, Griffiths, Tiffin.

10. John Thieme propose le terme « con-text » pour indiquer le Writing back et " pre-text » pour l'œuvre canonique. Pour un approfondissement voir Postcolonial Con-texts: Writing Back to the Canon. Literature, Culture and Identity, London, Continuum Press, 2001, pp.2-3

11. Les exemples plus célèbres sont : Wide Sargasso Sea de Jean Rhys qu'il s'agit du writing back de Jane Eyre de Charlotte Brontë, Foe de John Maxwell Coetzee (Robinson Crusoé de Daniel Defoe) ; Omeros de Derek Walcott (Odyssée de Homère).

12. Pour un approfondissement de cette triste et violente page d'histoire, voir le film documentaire «Kanesatake : 270 ans de résistance» de la réalisatrice autochtone Alanis Obomsawin.

13. Selon l'artiste: «Miss Chief Eagle Testickle, a character that appears in much of my work, was created as a way of challenging the subjectivity of the artist, challenging these signifiers of aboriginal identity that had become so popular in Hollywood film and nineteenth-century painting ». Voir, Kent Monkman, "Tonto Takes Charge. Miss Chief Eagle Testickle : a portrait », The Warlus Arts and Culture, 12/09/2012, [URL] https://thewalrus.ca/2008-05-detail/ [Consulté le 22/03/2018]

14. Lors d'une conférence livrée le 4 mars 2011 à la Galerie Leonard \& Bina Ellen de Concordia University à Montréal, l'artiste a déclaré d'avoir été inspiré par la chanteuse Cher et par sa période «half breed» car elle était à la fois glamour et sexuellement ambigüe. Information reportée par Michèle Thériault (dir.), Interpellations : three essays on Kent Monkman, Montréal, : Galerie Leonard \& Bina Ellen Art Gallery, 2012, p.42

15. On préfère l'emploi du terme anglais « Two Spirit » en solidarité avec la cause autochtone. Depuis les années 1990, les Autochtones découragent l'utilisation de terme berdache étant disqualifiant et inapproprié. Le terme péjoratif «berdache », dérivant du persan bardaj, désigne en français un jeune homosexuel passif. Pour un approfondissement de l'étymologie du mot « berdache » : Guy Ménard « Du berdache au Berdache : lectures de l'homosexualité dans la culture québécoise " Anthropologie et Sociétés, n 93, 1985, pp.115-138 [URL] http://www.erudit.org/fr/ revues/as/1985-v9-n3-as509/006292ar/ [Consulté le 22/04/2017] ; Laurence Hérault, " Transgression et désordre dans le genre : les explorateurs français aux prises avec les “ berdaches " amérindiens ", Etnográfica, vol. XIV, n², 2010 [URL] http:// etnografica.revues.org/316 [Consulté le 22/04/2017]

16. Ici on fait référence au texte de Perry Miller « Errand into the Wilderness ».

17. Pour en donner quelques exemples : The Rocky Mountains, Lander's Peak (1863), Merced River, Yosemite Valley (1866), Emigrants Crossing the Plains - The Oregon Trail (1869), et Indians Spear Fishing 
(1862). Pour un approfondissement du mythe de la « Wilderness » voir Roderick Nash Wilderness and the American Mind, Yale, 1982 et Angela Miller The Empire of the Eye, Ithaca, Cornell University Press, 1991.

18. Voir à ce sujet le site du Parc National de la Vallée Yosemite, https://www.nps.gov/yose/ learn/historyculture/indian-village-of-the-ahwahnee.htm

19. Pour un approfondissement, voir tout le premier chapitre «Territoires superposés, histoires enchevêtrées ». La violence géographique et la dépossession du territoire occupent une place fondamentale dans la pensée Saïdienne. Il faut tenir compte du fait que Saïd, étant un Palestinien né sous mandat britannique, était lui-même un dépossédé. À ce propos, voir l'introduction, p. 30. 20. A l'heure actuelle, les Autochtones nord-américains réclament aussi leurs territoires physiques, mais la question est loin d'être réglée.

21. Comme il l'affirme : "Se faire reconnaître, c'est recartographier puis occuper la place réservée à la subordination dans les formes culturelles impériales, 1 'occuper en toute conscience de soi, se battre pour elle sur le même territoire autrefois régi par une conscience qui postulait la subordination d'un Autre étiqueté inférieur. D'où réinscription. " [Italique dans le texte]. Edward W. Saïd, Op. cit. p.301

La « réinscription » fait partie du processus de décolonisation culturelle qui Saïd appelle « voyage de pénétration ». Il faut pénétrer au « cœur des ténèbres » du discours de l'Europe occidentale, s'y mêler pour le transformer et de lui faire reconnaître les histoires marginalisées, réprimées et oubliées. Voir à ce sujet p. 309 et tout le paragraphe p.337

22. Comme nous l'explique l'artiste: "Miss Chief is this playful way of inserting ourselves into these painted histories where we didn't exist or were sidelined as bit players ». Ibidem

23. Pour une définition du style Camp voir, Susan Sontag, " Notes on 'Camp' »", Against interpretation and other essays, Londres, Penguin, 2009, pp.275-292. Article disponible également en ligne, http://core.roehampton.ac.uk/repository2/content2/subs/d.steedman/d.steedman1608/ Sontag\%20(2009)\%20Notes\%20on\%20Camp.pdf [Consulté le 23/04/2017].

24. Les collections des statues africaines qui devaient constituer la base du Musée de l'Homme à Paris ont été la source d'inspiration de Les Demoiselles d'Avignon (1907). Voir à ce sujet Dominique Legros, «Les Demoiselles d'Avignon de Picasso et les pièces africaines contemporaines du tableau : Esthétique émancipatrice ou répressive ?» Sociologie et sociétés $\mathrm{n}^{\circ} 172,1985, \mathrm{pp} .71-82$. [URL] http://id.erudit.org/iderudit/001426ar [Consulté le 23/04/2017].

C'est intéressant de remarquer que Picasso a été largement contesté et parodié par nombreux artistes y compris Jean-Michel Basquiat qui lors d'une interview a affirmé : « Picasso est venu à l'Art primitif pour redonner ses lettres de noblesse à l'Art Occidental et moi je suis venu à Picasso pour donner ses lettres de noblesse à l'Art dit primitif. » Cité par Alexis de Vanssay en Jean-Michel Basquiat : The Radiant Child [En ligne] http://www.iletaitunefoislecinema.com/critique/3953/ jeanmichel-basquiat-the-radiant-child

25. Selon Bill Ashcroft et all. ces objets d'art ont été perçus par les occidentaux comme des: "échantillons de cultures «conservées dans le temps" et issues d'impulsions primitives communes à tous les hommes. [...] Cependant, dès le début, cette conception ethnographique s'est accompagnée d'une vision plus radicale, angoissante et complexe selon laquelle l'art primitif était vu comme exprimant "l'autre face » de la psyché européenne civilisée, la face sombre de l'homme. Telle est la peur exprimée dans des œuvres comme The Heart of Darkness [Au cœur des ténèbres] de Joseph Conrad ». Op. cit. p. 186.

26. Voir à ce sujet la peinture Trappers of Men (2006).

27. Voir à ce propos les « œuvres de réponse » à Curtis des artistes photographes autochtones Zig Jackson, Wendy Red Star, Terrance Houle et Will Wilson.

28. Il s'agit d'une autocitation, voir la performance associée Taxonomy of the European Male (2005). 


\section{RÉSUMÉS}

À la suite d'une série d'évènements historiques de grande relevance sociopolitique, les années 1990 ont connu une montée d'initiatives menées par les artistes autochtones nord-américains visant à une récupération et une divulgation de leur l'héritage culturel, et, de ce fait, à une revendication de leur visibilité dans le panorama international. De cette résurgence, l'artiste multimédial autochtone canadien, Kent Monkman, constitue l'une des voix plus actives et plus emblématiques. En créant un dialogue épistémologique entre les études postcoloniales et l'art autochtone, cet article interrogera l'œuvre de Kent Monkman au prisme d'une notion dont sera proposée une définition, à savoir le « Painting Back ».

After a series of significant socio-political events, the 1990s witnessed a growth of initiatives led by Native North American artists and aimed at repossessing and disclosing their cultural heritage, and thus claiming their international visibility. One of the most active and iconic spokesmen of this resurgence was, and still being, Canadian First Nations multimedia artist, Kent Monkman.

Creating an epistemological dialogue between Postcolonial Studies and Native Art, this paper will examine the oeuvre of Kent Monkman through the prism of "Painting Back" whose definition will be proposed in the following pages.

\section{INDEX}

Keywords : counter-narrative; native art; dispossession; cultural reappropriation; Kent Monkman; representation; painting back

Mots-clés : contre-discours ; art autochtone ; dépossession ; réappropriation culturelle ; Kent Monkman; représentation; painting back.

\section{AUTEUR}

\section{VALENTINA PANCALDI}

Doctorante en Littérature Comparée. Dép. Littératures et langues du monde. Université de Montréal, QC, Canada en cotutelle avec l'Université Sorbonne Nouvelle. Dép. Littérature Générale et Comparée, Paris, France) 